\title{
Characterizing Infrastructure Resilience in Disasters Using Dynamic Network Analysis of Consumers' Service Disruption Patterns
}

\author{
Hadi Nazarnia ${ }^{a^{*}}$, Hadi Sarmasti ${ }^{b}$ \\ ${ }^{a}$ Department of Civil and Environmental Engineering, Florida International University, Miami, USA. \\ ${ }^{b}$ Ph.D. Candidate, Department of Civil Engineering, Sahand University of Technology, Tabriz, Iran.
}

Received 03 June 2018; Accepted 03 September 2018

\begin{abstract}
This study proposes a network analysis framework for characterizing infrastructure resilience in the aftermath of disasters through the use of consumers' service disruption information. In the presented framework, the notion of "peers" is used to construct the network models of consumers experiencing service disruption in the aftermath of a disaster to understand the type and extent of infrastructure damages and specify disruption patterns. Data related to electricity disruption in Bhaktapur Nepal, in the aftermath of the 2015 Gorkha Earthquake is used to construct the network models of consumers' networks at different points in time in the aftermath of the disaster. The created models are then used in network analysis for examining the network topological characteristics (such as clustering) and specifying the attributes of service disruption. The contribution of this paper lie in: (i) the development and validation of a novel network from disruption information, (ii) identify the extent of infrastructure disruption, type of damage, and recoverability from changes in network topology over time.
\end{abstract}

Keywords: Disaster; Resilience; Infrastructure Systems; Network Analysis; 2015 Gorkha Earthquake.

\section{Introduction}

Resilience of Civil Infrastructure Systems (CISs) plays a crucial role in ensuring the viability of societies and performing the functions of daily activities, especially during extreme events and post disaster periods, with the aim of minimizing the adverse effects of disasters. Everyday life relies on the operation of different CISs, such as electric power, water and gas distribution, and transportation infrastructures. Disasters (such as Hurricane Sandy 2012, Hurricane Harvey 2017, or Northeast Blackout 2003) have shown vulnerability of civil infrastructures and emerged importance of having a resilient infrastructure. Recent planning work have focused on response and restoration and importance of this dimension of resilience, recoverability [1]. Despite the pervasive interest in disaster resilience assessment, risk assessment, network analysis, and vulnerability (disruption) of infrastructure systems, existing literature exhibit a lack of clarity in consideration of service disruption patterns and status of end-users in the assessment of the resiliency in CISs and mainly emphasis on components in the network. In addition, in an effort to evaluate the resilience of the CISs, scholars have tried to quantify the disruption period, response and recovery times and damages to the physical infrastructure networks, while striving to simplify the existing networks, which may lead to a number of errors in quantifying the disaster resilience of a CIS [2]. To address these issues and capture the real behavior of the CIS, the disruption characteristics of the infrastructure networks are implemented to assess the resilience of the infrastructure systems. This approach not only offers new insight regarding understanding the resilience of infrastructure and communities, but it also resolves the associated obstacles when obtaining data to create networks after any disruptive

* Corresponding author: hnaza001@fiu.edu

\section{do) http://dx.doi.org/10.28991/cej-03091165}

$>$ This is an open access article under the CC-BY license (https://creativecommons.org/licenses/by/4.0/).

(C) Authors retain all copyrights. 
event. This paper presents a novel methodology through the application of network analysis to mainly investigate different possible disruption patterns and to provide a new insight into the dimensions of infrastructure resilience that suffer damages after natural or man-made disaster. In addition, data of the devastating earthquake in Nepal in 2015 has been used to validate the model. In other words, the objective of this study is to create and test an integrated model to evaluate the resilience of the infrastructures that are comprised of social networks. The application of the framework is illustrated in an actual case study associated with the electricity infrastructure network of Bhaktapur, Nepal and the disruption-based model is able to model the behavior of the CIS properly. In the proposed framework, different patterns of disruption are obtained by Monte Carlo simulation to evaluate the characteristics of the system. The model measures important features of the resilient infrastructure network and does not require detailed information on the system, while it is difficult to acquire. The results show that the changes in the network topology over time provide insights into the extent of infrastructure disruption (i.e., sensitivity), type of damages (i.e., local vs. system-wide damages), and the recoverability of the infrastructure. Hence, the proposed network analysis framework creates a new computational approach that, in the absence of data related to physical infrastructure damages, enables the characterization of infrastructure resilience and disruption patterns based on consumer service status data.

\section{Background}

The societal need for uninterrupted performance of CISs has resulted in a significant surge in the number of publications on the resilience of CISs. However, despite substantial advances, assessing the resilience of CISs and its approaches remain challenging. CISs are composed of visible and invisible elements and parameters add complexity to the network. Such elements and parameters creates interdependency and complex socio-economic parameters, as well as several other influential parameters, in the function of the civil infrastructures [3].

Scholars have utilized several approaches to study the resilience of the CISs, all of which can be divided into two broad categories of qualitative and quantitative resilience assessment [4-7]. Among the quantitative approaches, one robust method is the network analysis approach. There are many reasons to justify the application of network analysis in the infrastructure system assessment. First of all the network has been used to represent many man-made, social, and biological systems, such as transportation infrastructures and underground water or sewer systems. For instance, in urban studies, the network approach has been applied to evaluate the traffic flow and land use changes [8]. Secondly, infrastructure networks are considered to be supply networks, the resilience of which is defined as the potential for remaining operational under any disruption and preserving its connectedness despite structural or functional losses [9]. That is why the analysis of the infrastructure resilience using different methods of network analysis is inevitable. These reasons, as well as several other studies on the assessment of infrastructural systems, confirm that the network method can be considered a strong tool in the study of CISs [5, 7, 10-15].

Researchers concur that networks can exhibit the interaction of systems, and such networks can be used to monitor, and control systems $[16,17]$. Hence, the network approach is a powerful tool in better understanding CISs and complex systems. Networks are shown as a graph in mathematical format. Generally, graph theory, as a comprehensive mathematical tool, has been used to investigate the characterizations of the infrastructure system and human interactions [46]. For example, Chai et al. 2011 highlighted the vulnerability of various infrastructures with principles of the social network analysis. The researchers remarked that the interdependency of the infrastructures is similar to the interaction of the individuals in society and prioritized the oil and gas infrastructure as the most important infrastructure, which led to the cascading failure of the entire system [18]. Eisenberg et al. (2017) combined social network analysis and power grid network analysis in South Korea to provide new insight of organizational inclusion with blackout risk. To do this, they utilized different measures of network analysis [19]. Yazdani et al. (2011) developed the water distribution system as a graph with nodes and physical links, which they used to examine several resiliency components; namely reliability, efficiency, and robustness. To quantify the resilience of the water infrastructure network, they applied some of the network theory metrics, not only to evaluate the existing network, but also to increase the redundancy of water networks in a time of network expansion [13, 14]. However, simplifications on these papers were not evitable. Furthermore, there are numerous parameters affecting the resilience of CISs. For example, human-infrastructure coupling or interdependency of the civil infrastructure [20]. Also, the disruption status of the end-users has not received much attention and the author believes that it might be a new tool on characterizing the resilience of the community with consideration of most of the effective criteria. In this paper, the measure of clustering in network analysis has been used to characterize the resilience of the CISs and to learn the possible disruption patterns in any disaster.

Generally, the network based approach can be divided into two main categories, depending on whether the particle flow is modeling on the infrastructure network or not: Topology-based approach and flow-based approach.

Topology-Based Network Analysis (Topological): In this approach, infrastructures are solely modeled based on their topologies. Their components consist of several nodes and links that have two discrete states: "operating" or "failure". Moreover, this model can be analyzed through the analytical or simulation approaches. The drawback with the analytical approach is a lack of consideration for the heterogeneity of nodes; meaning that, all nodes, such as source, 
transmission, and sink nodes are all regarded as the same. On the other hand, the simulation-based method takes node heterogeneity into account and applies it to the modeling process. It should be noted that there are many measures in the network science, some of the most applicable of which, such as the number of operating or failed elements, the inverse characteristic path length, the connectivity loss, and the redundancy ratio, are employed to evaluate the model. In addition, a topology-based approach obtains the topological features of the infrastructure's critical components before a disaster and is able to suggest a number of improvement tasks to increase the robustness of the network.

Flow-Based Network Analysis (Functional): This approach constitutes nodes and edges which have the capacity of loads. Compared to the topological method, the functional method requires excessive computational analyses. Although this feature harnesses the opportunity to assess the impact of the disruption of the catastrophes, it requires more information about the system and usually yields different results from the topology-based approach [1, 21, 22].

Going back to the first model, topology modeling has the capacity to evaluate different aspects of the network, such as performance, security, traffic modeling and engineering, protocol development and analysis, as well as assessing numerous other algorithms [23]. Additionally, several studies have tried to show the crucial role of topology assessment in the characterization of power transmission systems. In fact, these models are the simplified versions of the real networks at the time of creation. This simplicity is the basis for many types of research; however, this practice has not been without a few drawbacks. Some scholars contest that, although infrastructure topology captures the system's functionality, it is not able to analyze the behavioral characteristics of the infrastructure systems on its own, and in some cases, it even misleads the decision makers about the situation of the infrastructures [24]. Although the importance of the topology-based approaches is not deniable, they are not able to demonstrate several features of the infrastructure network (e.g. capacity to respond, sensitivity or disruption pattern). Furthermore, different approaches for the analytical evaluation of resiliency need in-depth details of the network components and system features, which is not always feasible to acquire; since, due to confidentiality issues and antitrust laws, there is limited accessibility to information regarding physical damages to the system in the aftermath of any disaster [13, 14, 21, 24-26]. To address the limitation associated with the network-based approaches in assessing the resilience of CISs, disruption status of end-users has been used in the proposed framework to create a proper network that demonstrates the behavior of the civil infrastructure.

The present paper mainly focuses on the resilience of civil infrastructure networks, contributing to possible disruption patterns with simulation. Graph theory is a beneficial tool for understanding network resilience; however, the application of a number of hybrid approaches can be extremely fruitful in this matter. For instance, Winkler et al. (2011) presented a hybrid assessment methodology to evaluate the interdependencies among the infrastructure. They connect the physical fragility model with the topology of the actual connections between infrastructures using some of the network measures, such as betweenness, clustering, node degree and Euclidean distance, to assess the failure propagation in the networks. Others have coupled network-based methods with the structural reliability theory to evaluate the seismic performance of infrastructure systems [27]. Although all of these methods have shed more light over the resilience of civil infrastructures, the methods require detailed information of data to be created. In this paper, the characteristics of the disruption and resilience of the CISs will be evaluated with regard to a few measures of the social network topology. The proposed framework borrows several concepts from sociology (i.e., peer) to form the network from the service status of end users in the system. These components of the models create a network similar to a social network. The resilience of networks is evaluated in a variety of ways, including network performance and connectivity analysis. Gao et al 2016 characterize system's state with the average nearest-neighbor activity and discuss it with outgoing and ingoing weighted degree [4]. By utilizing the measures of network analysis, such as clustering coefficient, the designed network provides a number of important measures and elements of resilience, recoverability, and type of perturbation in the system, in a CIS. Other measures of the network analysis, such as density, degree centrality or modularity, are beyond the scope of this paper and need more contemplation; these measures may provide more insight toward the resiliency of CISs.

\section{Materials and Methods}

To study infrastructure network resiliency, various data regarding the failures in the network components and damages to the civil infrastructure is required; for instance, the spatial information of the damages, type of the damaged and undamaged components, and the connectivity of the components (topology of the network). This required information to conduct a topology-based analysis of infrastructure networks is difficult to acquire in most cases because in the post disaster response period, time plays a key role in saving the inhabitants' lives and a major concern is to rescue the injured in a timely manner. In addition, the outcomes of topology-based models mainly provide insights regarding the vulnerability of networks. Modeling also enables the identification of critical nodes in a network. Hence, topologybased network models are more useful in the evaluation of the serviceability of infrastructure networks prior to any disasters. For a post-disaster study, collecting data regarding disruption in infrastructure services provides critical information. Most of the existing studies utilize the extent of service disruption (e.g., the number of households that experienced electricity disruption) and the speed of restoration (i.e., the duration of service disruption). Further 
additional insights can be learned by studying the disruption in infrastructure networks; however, the lack of methodological approaches has hindered the analysis of disruption patterns in this area.

To address this gap and provide a means for a better understanding of disruption in infrastructure networks, this study proposes a novel methodology based on the theoretical underpinnings of social network analysis and uses the feature of network topology and measures of network analysis. According to social network theories, two nodes in a network are considered to be "peers" if the nodes have a similar status, have social attributes, or communicate and work with each other. Based on the same premise from the social network, in the proposed methodology, infrastructure users can be considered as "peers" if they have a similar status in terms of access to infrastructure services. Based on this fundamental premise, infrastructure users are considered as nodes and their connectivity is modeled with links according to the status of their infrastructure services and disruption at different points in time in the aftermath of a disaster. Then, through the analysis of the structure of the social network of infrastructure users, disruption patterns and the behavior of infrastructure networks are examined. The proposed methodology is capable of providing insights about a network's sensitivity to disruption, service disruption patterns, and the capacity of the infrastructure network to respond without the need for information related to the age and dependencies of physical infrastructure.

As shown in Figure 1, the proposed methodology for the assessment of service disruption in infrastructure networks is composed of five steps:

1. Determine the infrastructure service state.

2. Create the adjacency matrix for social network at different time intervals.

3. Model the networks at different time intervals (social network).

4. Examine the characteristics of infrastructure disruption.

5. Conduct the trend analysis over time in social network (Analysis of the changes).

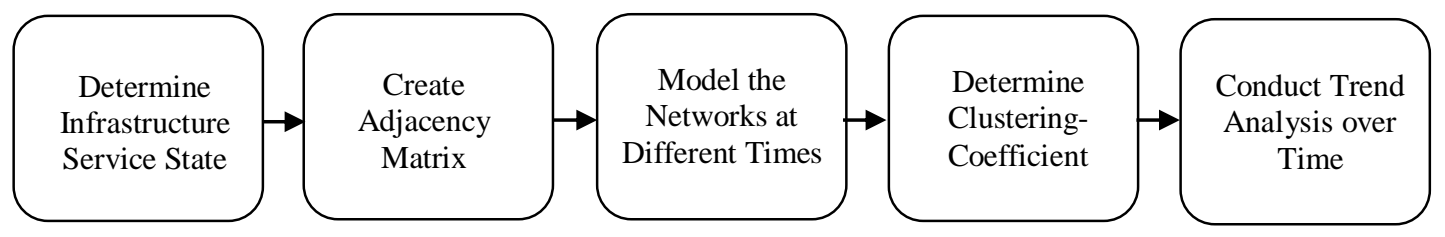

Figure 1. Framework for disruption based model

\subsection{Develop Social Network Based on the Infrastructure Disruption (Step1-3)}

The first step in the proposed framework is to define peer groups related to infrastructure service disruption at different points in time. The model has been inspired by the notion of peer groups and the similarity-based approach in the social network. Link prediction is a significant concern in many areas and many attempts have been made to measure the probability of existing links between two nodes. The basic assumption of these methods is the similarity of the two nodes, which leads to a link between them. This concept has been applied to social network analysis and biology to predict the interaction of the individuals or proteins [28]. In social network analysis, people who live in a specific neighborhood, students in a class, or children with the same age are more likely to have the same features. Peer group structure in the social sciences refers to the pattern of interaction among adolescents. Accordingly, the similarity of the individuals is used to understand the interaction between the peers and to interpret the pattern of relationships among them for a number of years. The peer group is one of the important concepts in social network analysis, which evaluates the connectivity of the individuals based on their similarities in behavior, status, or characteristics. Based on the same premise from social sciences, in the proposed framework, peers are defined as infrastructure users who have the same service status in the aftermath of a disaster; for example, users who have experienced electricity disruption at time $t$.

To build a social network associated with a specific infrastructure service, the adjacency matrix of the network should be abstracted. To do this, the information related to the status of infrastructure services for different users should be collected. As shown in table 1 and figure 2, in the creation of the adjacency matrix, the state of service for a user can be either one (service exists) or zero (service is disrupted) at time $t$. If two users experience service disruption at time $t$, they are considered as peers and the two users are connected to each other.

Table 1. Adjacency matrix of Sam and Jack

\begin{tabular}{cc}
\hline Adjacency matrix & User 2 \\
User 1 & 1 or 0 \\
\hline
\end{tabular}


(1)

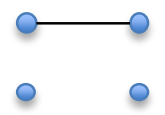

Figure 2. Connection between two nodes

The state of infrastructure services for each node or user can be defined as a binary variable in the following format:

$S_{i}(t)$ : State of user $i$ at time $\mathrm{t}$

$\mathrm{j}=$ number of various households

$S_{j}(t)$ : State of user $j$ at time $\mathrm{t}$

$S_{i}(t)$ or $S_{j}(t)= \begin{cases}1 & \text { If infrastructure service exist } \\ 0 & \text { If infratsructure service is disrupted }\end{cases}$

Using the state of infrastructure services for different users, the mathematical representation of the network is determined based on a graph $\mathrm{G}(\mathrm{V}, \mathrm{E})$. Generally graph is defined by a set of vertices $\mathrm{V}$ (or nodes) and by a set of an unordered pair of different edges E (or links). If a link exists between two nodes "i" and " $\mathrm{j}$ ", these nodes are connected. The link between nodes " $\mathrm{i}$ " and " $\mathrm{j}$ " is represented by $(\mathrm{i}, \mathrm{j})$. In the proposed framework, the value of $(\mathrm{i}, \mathrm{j})$ is determined using Equation 2:

$$
\text { If } S_{i}(t)=S_{j}(t)=0 ; \text { Then }(i, j)=1 ; \text { Otherwise }(i, j)=0
$$

In the framework, the networks are an undirected and unweighted graph represented by adjacency matrix R, which is a square matrix. $(j, j)$ determines the links in the adjacency matrix. The adjacency matrix is symmetric. All diagonal elements of the matrix are equal to zero because there is no self-loop in the network.

Application of this concept will be explained through a simple example. Let us consider a scenario in which four infrastructure users exist: Sam, Jack, Michael, and Sara $\left(\mathrm{U}_{1}\right.$ is Sam, $\mathrm{U}_{2}$ is Jack, $\mathrm{U}_{3}$ is Michael, and $\mathrm{U}_{4}$ is Sara). A storm has occurred and caused electricity disruption in these infrastructure users. If Sam and Jack do not have electricity in their house at time $t_{1}$ (one day after the storm), their infrastructure service state will be 1 , and thus these two users are connected with a link (Figure 3 ) and the value of $\left(\mathrm{U}_{1}, \mathrm{U}_{2}\right)$ in the adjacency matrix will be 1 (Table 1 ).

Table 2. Adjacency matrix of Sam and Jack

\begin{tabular}{|c|c|c|}
\hline Connection & Household 1 & Household 2 \\
\hline Household 1 & 0 & 1 \\
\hline Household 2 & 1 & 0 \\
\hline
\end{tabular}

Figure 3. Network structure of $U_{1}$ and $U_{2}$ in time $t_{2}$

In the aftermath of a disaster, disrupted infrastructure services are gradually restored. Accordingly, the infrastructure service states for each user change, and thus the peer links between users change. In the previous example, if all the users experience electricity disruption in the aftermath of the storm $\left(\mathrm{t}_{1}\right)$ (Table 3$)$, their infrastructure service state will be the same (Table 4), and hence all the nodes will be connected to each other, as shown in Figure 4. A few days after the storm $\left(\mathrm{t}_{2}\right)$, electricity service is not restored for any of the users. Hence, the network links remain the same as $t_{1}$. However, after a week $\left(\mathrm{t}_{3}\right)$, electricity service is restored for Sam's house. Hence, Sam's service state changes to 0, and thus, the links between Sam's node and other users are removed (Sam is no longer considered as a peer to the other users), as shown in Figure 5.

Table 3. Power outage for different users is the basis for creating social network (time 1-2)

\begin{tabular}{ccccc}
\hline Power Outage & Sam & Jack & Michael & Sara \\
\hline Time 1 & Yes & Yes & Yes & Yes \\
Time 2 & Yes & Yes & Yes & Yes \\
\hline
\end{tabular}


Table 4. Adjacency matrix of users in a time of $t_{1}, t_{2}$ after the disruption

\begin{tabular}{c|cccc}
\hline Adjacency matrix & Sam & Jack & Michael & Sara \\
\hline Sam & 0 & 1 & 1 & 1 \\
Jack & 1 & 0 & 1 & 1 \\
Michael & 1 & 1 & 0 & 1 \\
Sara & 1 & 1 & 1 & 0 \\
\hline
\end{tabular}

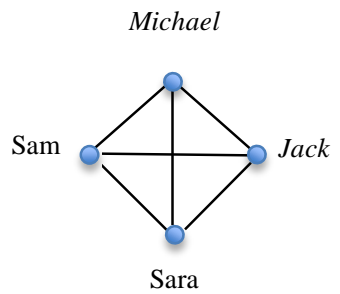

Figure 4. Network structure of users in a time of $t_{1}, t_{2}$ after the disruption

Table 5. Power outage for different users is the basis for creating social network (time 1-2-3)

\begin{tabular}{ccccc}
\hline Power Outage & Sam & Jack & Michael & Sara \\
\hline Time 1 & Yes & Yes & Yes & Yes \\
Time 2 & Yes & Yes & Yes & Yes \\
Time 3 & No & Yes & Yes & Yes \\
\hline
\end{tabular}

Table 6. Adjacency matrix of users in a time of $t_{3}$ after the disruption

\begin{tabular}{c|cccc}
\hline Adjacency matrix & Sam & Jack & Michael & Sara \\
\hline Sam & 0 & 0 & 0 & 0 \\
Jack & 0 & 0 & 1 & 1 \\
Michael & 0 & 1 & 0 & 1 \\
Sara & 0 & 1 & 1 & 0 \\
\hline & Michael & & \\
& Sam & Sara & & \\
& & &
\end{tabular}

Figure 5. Network structure of users in a time of $t_{3}$ after the disruption

\subsection{Examine the Characteristics of Infrastructure Disruption (Step 4)}

The objective of the fourth step is to examine the characteristics of infrastructure service disruption through the use of appropriate measures of social network analysis. Different network measures provide different insights regarding the characteristics of a network. For instance, in 2016, Wu et al. used the clustering coefficient measure of social networks to predict the possible links and provide new insights in this field. The main incentive for them was the triangle information, triangles formed by common-neighbors, and measure of the clustering coefficient, which demonstrates the density of the triangles. They highlighted the importance of the clustering coefficient in linking the same structural position in nodes [29]. In fact, the clustering coefficient is defined as a measure of the probability for a link to exist between any two neighbors of a node; in other words, clustering coefficient is a measure which quantifies the connectivity of the neighbor nodes $[16,28,29,46]$. In the scope of this study, the objective is to examine the extent and type of infrastructure service disruption and to provide a novel practice to assess the resilience of CISs. In other words, in a disruption social network, if all the nodes are connected, this implies an extensive service disruption and vice versa. Also, if a certain number of nodes are connected and the other nodes are not, this implies service disruption in part of the infrastructure network. Hence, the clustering behavior of the nodes in the disruption social network can provide useful insights about the patterns of service disruption in the infrastructure. To this end, the average clustering coefficient measure is used for evaluating clustering behaviors in the disruption social network. 
Clustering is a network property which demonstrates a deviation from random network behaviors. Neighborhood notion is the basis of the clustering quantification. From a graph-theoretical aspect, clustering shows the presence of a high number of triangles in the network; in other words, the relative number of three vertices that are connected to each of the other vertices. The clustering coefficient in a network can be determined using Equation 3.

$$
\mathrm{C}=\frac{3 \text { (number of traingles in the network) }}{\text { number of the connected triples of vertices }}
$$

To restrict the clustering coefficient in the boundary of $[0,1]$, factor of 3 adjusts the denominator of the equation because each vertices of a triangle contributes to three triplets. These triplets may create a triangle and the clustering coefficient depicts the proportion of all triplets that has a third edge to create a triangle. The ratio of means is more popular than the mean of ratio in analytical evaluations. Mean of ratio approach is widely utilized for data analysis and research and has been used to calculate the aforementioned coefficient in equation 3.

\subsection{Conduct the Trend Analysis over the Time in Social Network (Step 5)}

To evaluate the characteristics of infrastructure service disruption and the resilience, in this step, the clustering coefficient of different social networks is drawn versus time. If the infrastructure services experience a disruption in the aftermath of a disaster, the clustering coefficient is obtained based on the explanation. For instance, in the previous example, all end users (i.e., $\mathrm{U}_{1-4}$ ) have a blackout in time 1 and 2. Consequently, all of the users participate in social network creation and therefore, the associated clustering coefficients for this period are equal to one. However, when user 1 retrieves the electricity service (in time $t_{3}$ ), it is no longer considered a peer and the related links of that user are removed from the social network. Consequently, the clustering coefficient decreases to a number between zero and one.

The remaining part of this section discusses three major characteristics of the infrastructure disruption:

(i) Sensitivity

(ii) Local or System-Wide Disruption

(iii) Recoverability

(i) Sensitivity: The notion of sensitivity has been presented in multiple formats in the literature. According to Adger 2006 and Gallopin 2003, sensitivity is defined as the amount of absorbed impacts by a system without confronting the possibility of a long term and significant disturbance and failure; in other words, sensitivity is considered as a degree of changes in the system after an external or internal disturbance [30]. Some scholars contend that sensitivity is one of the most significant criteria to assess the infrastructure resiliency. As an example, Gallopin (2006) proposes a conceptual model that constitutes sensitivity as a tool to examine resiliency [31]. Sensitivity has also been combined with other parameters, such as exposure, and has been presented as a system response to any external changes [32]. In addition, the sensitivity of infrastructure systems depends on: system condition, dependencies with other infrastructures [33], humaninfrastructure coupling, and the preparedness of organizations managing and operating these systems [20].

An increase in clustering coefficient results in an increase in the number of service users that experience disruption in the aftermath of a disaster. As a matter of fact, the clustering coefficient determines the density of the "peer" in the network. Hence, a system with a large clustering coefficient has more sensitivity and vice versa. Figure 6 shows the measure of sensitivity in two different systems. System 1 has a larger amount of sensitivity compared with system 2, which has a lower cluster coefficient. In this figure, the vertical axis represents the amount of disruption in the infrastructure system in the aftermath of a disaster and the horizontal axis demonstrates the variation of recovery time. Curve 1 shows more sensitivity to disturbance; therefore, based on the "peer" notion, the associated network is more congested and more service users lost infrastructure service. As a result, the entire infrastructure system has a higher clustering coefficient.

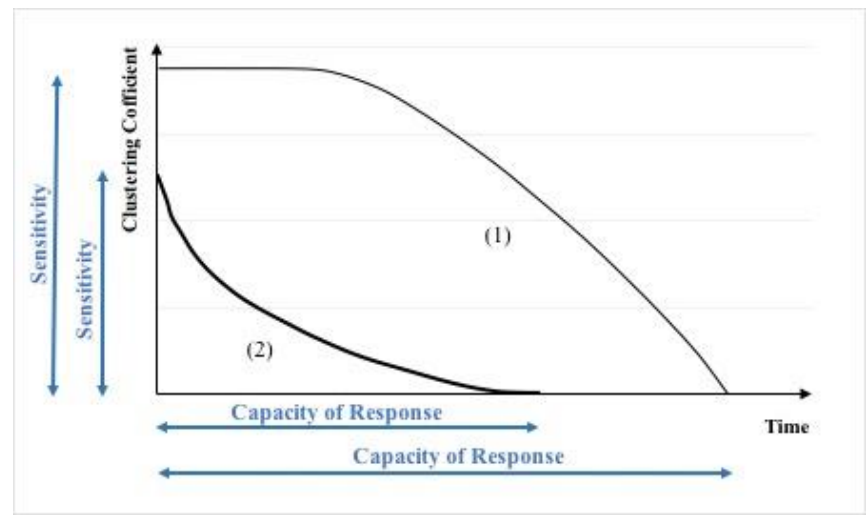

Figure 6. Sensitivity of system 1 / system 2 and capacity of response (recoverability) in different disruption patterns 
(ii) Local or System-Wide Disruption: Another important objective of this study is to examine the types of disruption in the infrastructure system. If all of the end users experience an outage in their service after a disruption, this connotes severe disruption in the system and there is a probability that the damage is related to the important components of the entire system, which works as a hub for the infrastructure network service. Accordingly, the major portion of the network loses service. Based on the proposed methodology, this condition has more connected peers; hence, a more congested network and a high clustering coefficient. On the other hand, if a certain number of users experience failure in their service, the infrastructure network encounters local disruption in the system and damages affiliated with lowlevel components of the system, which are most likely limited to a specific region; thus, the rate of change in clustering coefficient or slope of the curve is low.

In the previous example, at time $\mathrm{t}_{1,2}$ all users do not have access to electricity. Gradually, all users acquire service in the network and all links are removed, as is shown in Figure 7a, b. In this case, the associated clustering coefficient of the models are equivalent to one in the beginning and then become zero when all the users have access to electricity.

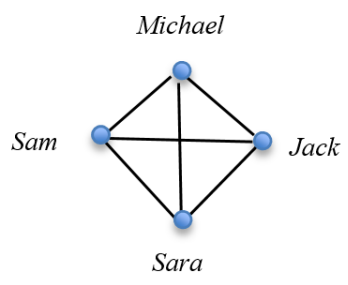

(a) $t_{1}$

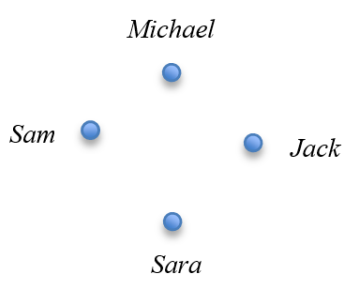

(b) $\mathrm{t}_{2}$

Figure 7. A network with system-wide disruption

Figure 8 presents a pattern of different types of disruption in the infrastructure network. As shown, the slope of the trend line demonstrates the rate of service recovery which shows the probability of system wide or local damages in the system. In a time of no changes in clustering coefficient, system still experiences outage and this disruption can be considered as a system wide. Also, if the rate of changes in clustering coefficient is high, it means the higher numbers of household retains their service, and it is called as a system-wide. In other words, huge changes in clustering coefficient indicates how fast system recovers and the links between disrupted households diminish. On the other hand, a decreased slope in the recovery process means the system needs more time to retain electricity; hence, the system exhibits local disruption and the rate of change of the clustering coefficient is low. As an example, the second portion of the curve 1 has a low rate of variation in clustering coefficient, which is associated with local disruption in the system; however, the first portion of the curve 1 remains steady and the value of the clustering coefficient is almost the same in this period. Another possible situation for system-wide disruption is when there is a significant amount of changes in the clustering coefficient in a short time. Likewise, Curve 2 shows this rapid variation in the clustering coefficient, which implies a decreased recovery time for the service of the infrastructure system.

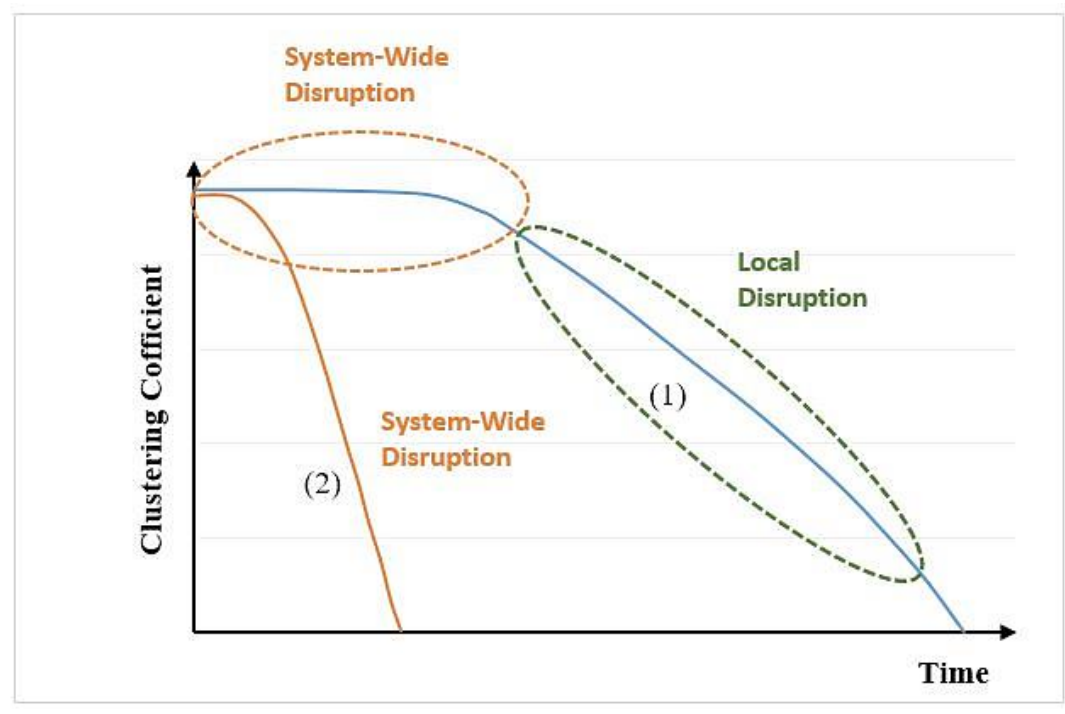

Figure 8. System-wide versus local disruption pattern

(iii) Recoverability or Rapidity: The third outcome of the proposed methodology and one of the resilience dimensions, is the capacity of response. The general definition of resiliency implies the ability of a system to recover from a disruption and the ability of the system to regain normal conditions after any disturbance. According to Bruneau 
and Reinhorn 2006, restoration of a specific system in a certain time period is one of the features of a resilient system. Accordingly, this feature of an infrastructure system plays a significant role in the aftermath of any disruption occurrence [33]. In the proposed model, the recoverability is contrary proportional to the time from the initial condition to the final condition, hence, after any disruption, less time to recovery shows more capacity of response in the system.

Based on the proposed methodology and the drawn figure for clustering coefficient versus time, the distance from the vertical axis demonstrates the ability of the infrastructure network to recover disconnected parts. In other words, the distance represents the time needed to return to the normal serviceability status. Less distance from the time origin (i.e., disaster) indicates a high recoverability response and a more resilient system. Figure 6 shows two different systems with two different capacities of response. System 1 compared to system 2 has a reduced recoverability and needs more time to obtain a normal service status.

\section{Simulation of Different Possible Disruption Patterns}

The power grid network comprises different elements, such as transmission lines with different voltages (e.g., 11, 66, $132 \mathrm{KV}$ ), generator substations, and distribution substations which receive and transmit power to the other substations or distribute power in local distribution grids. Of course, in a time of disaster, any damages to the components of the electric power infrastructure lead to different patterns of disruption in the system. For instance, damages to the generator substations of the city give rise to a blackout in the entire region and when the generator is fixed, large regions recover service instantly. On the other hand, damages in the distribution grids (e.g., falling poles) may result in the local perturbation, as is shown in Figure 9. In Bhaktapur, wires attached to the outside of buildings were damaged, which caused local disruption to the power grids and electricity networks $[35,36]$.
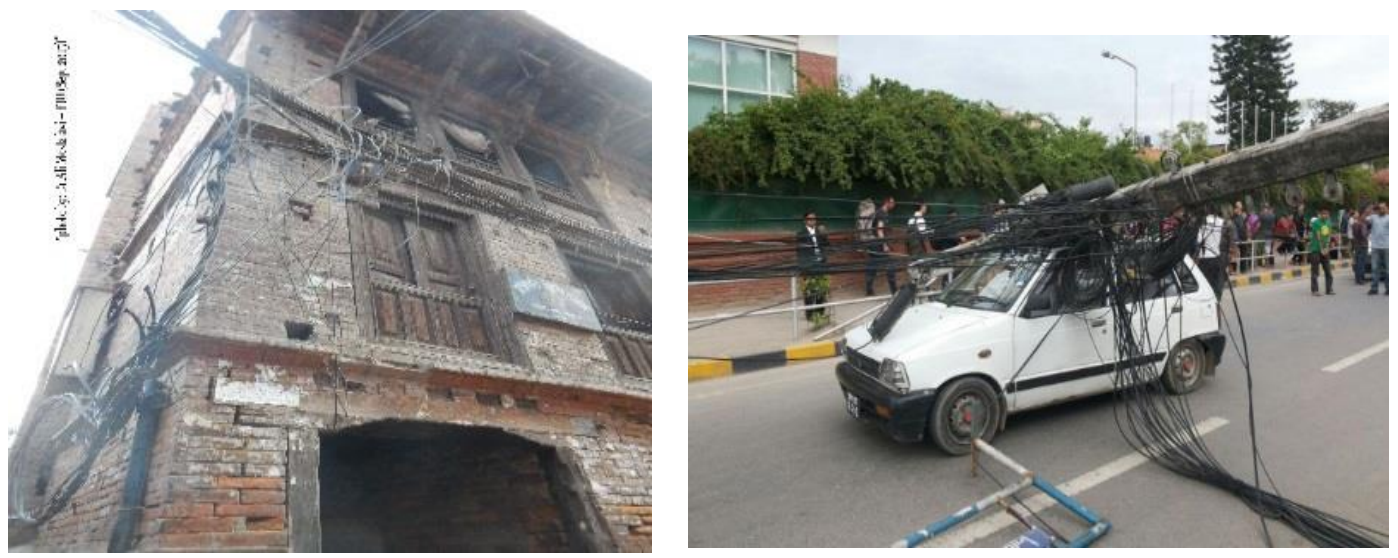

Figure 9. Locally damage in the electric power system due to attached wires to buildings pole drop [36]

To analyze different possible scenarios that might have occurred in random or targeted disruptive events, computer simulations have been used. A different pattern of the blackout is obtained in this way, as shown in Figure 11. Monte Carlo simulation is used to detect different patterns of possible disruption and to assess the various possible outcomes. The objective of the simulation is to confirm that this is not the only pattern of disruption in the aftermath of a disaster.

\subsection{Simulation Requirements}

An important component of the model in the simulation is to find the similarity between different nodes or users. In these simulations, the networks are created based on disruption to disruption assesse and characterize the CISs. To identify the peer in the network, each user or node of the network needs an attribute to be distinguished from other nodes. Based on this attribute, the node is compared with measures of local or system-wide disruption. To assign an attribute to the nodes, a random number is generated for each user. Consequently, the associated attribute determines the potential of nodes to be considered as peers and connect to other nodes. When the nodes have the same state of disrupted, the nodes are connected and contribute to forming a network.

The probability theory is applied to determine the measures of local or system-wide disruption in the simulation. If the probability of system-wide disruption is equal to (Pri) and the probability of the local disruption in the system $\left(\operatorname{Pr}_{\mathrm{j}}\right)$, the mathematical format of the steps to create the social network of disruption in different scenarios are as follows:

Probability ("Disruption | System-wide damage”) $=\operatorname{Pr}_{\mathrm{i}}$

Probability ("Disruption | Local damage") $=\operatorname{Pr}_{\mathrm{j}}$

The variables "i" and "j" refer to system-wide disruption and local disruption in the specific scenario, respectively. 
In this study, the probabilities of local disruption in different scenarios are considered to be $40 \%$ and $30 \%$, while the probabilities of system-wide disruption in different scenarios are $60 \%, 50 \%$, and $40 \%$. In other words, the probability of $40 \%$ implies that if the generated random number is less than 0.4 , that user or system experiences blackout.

Table 5 illustrates different scenarios with assigned probability for system-wide and local disruption in the network.

Table 7. Power outage for different users is the basis for creating social network (time 1-2)

\begin{tabular}{cccc}
\hline & \multicolumn{3}{c}{ Disruption Probability } \\
\hline & Scenario-1 & Scenario-2 & Scenario-3 \\
\cline { 2 - 4 } System-wide disruption $\left(P r_{i}\right)$ & $40 \%$ & $30 \%$ & $30 \%$ \\
Local disruption $\left(P r_{j}\right)$ & $60 \%$ & $50 \%$ & $40 \%$ \\
Associated figure & Figure 15a & Figure 15b & Figure 15c \\
\hline
\end{tabular}

A different network with different vulnerability at different times after the disruption would be developed in this approach. The following section provides a comprehensive explanation for the simulation.

\subsection{Simulation Algorithm}

This algorithm has been used to simulate the creation of social networks at different time intervals:

1. Generate a random number for the entire system and for each user.

2. Consider a different disruption probability for the system and the local situation. $\left(\operatorname{Pr}_{\mathrm{i}}\right.$ and $\left.\operatorname{Pr}_{\mathrm{j}}\right)$.

3. Assess the probability of disruption in the entire system.

4. Assess the probability of local disruption in the system (if there is no system-wide disruption).

5. Combine the system-wide and local disruption in the system.

The applied algorithm is presented in Figure 14 briefly and the rest of the process is the same as the previously built model in the methodology and case study.

Assess the Probability of Disruption in the Entire System: To start the simulation, a random number is generated for the entire system and compared with the selected $\operatorname{Pr}_{i}$; if the random number is less than $\operatorname{Pr}_{i}$, the state of the entire system is "out of service or disrupted" and all the elements (except diagonal elements) in the adjacency matrix are equal to one. If the random number is more than the selected percent, the probability of damages is low, the system is probably not disrupted, and the elements of the adjacency matrix for the entire system are equal to zero. In the case that the entire system does not have service, the local disruption would not be examined because there is already an outage in the system.

Assess the Probability of Local Disruption in the System: When the entire system does not experience damage, there is a possibility of disruption in the different local areas of the network during the recovery period. To check the local damage, distinct random numbers are generated for each node (user) in a different point in time after the disaster occurred. At a local level, if the random number of each node is less than $\operatorname{Pr}_{\mathrm{j}}$, the node is connected to another and accordingly, the associated states are equal to one. In addition, the probability of continuing disruption is examined in the subsequent time if the user does not have electricity at a given time. The simulation assumes that if the user obtains electric power, then the user would not experience a power outage during the recovery time.

Combine the System-Wide and Local Disruption in the System: After assessing the possibility of disruption in the entire system and at the local level, the holistic social network is retrieved based on the following approach:

Connect all the nodes based on the disruption state in the whole system; otherwise, connect locally disrupted nodes and then decide the connectivity to create one network for each point in time. Figure 14 illustrates the applied flowchart for creating a social network for simulating different patterns of disruption in the infrastructure system. 


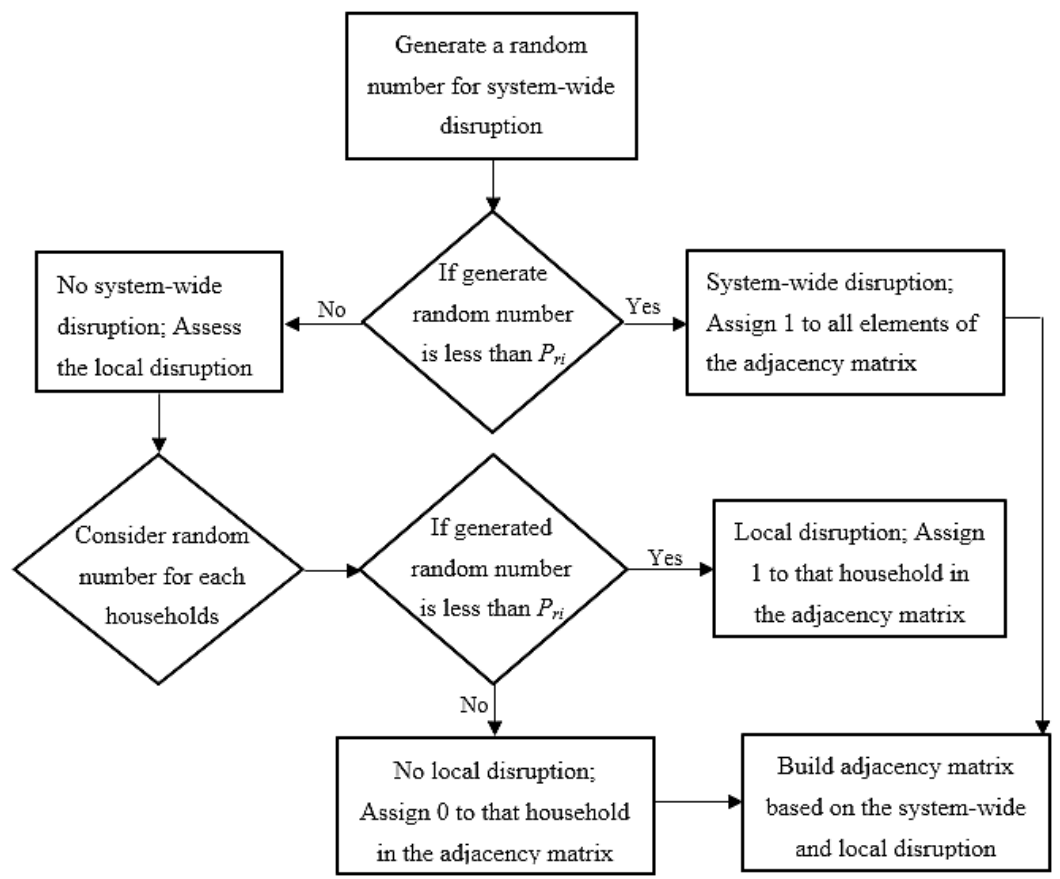

Figure 10. Flowchart of developing different scenarios in disruption

\subsection{Evaluate the disruption alternatives for different scenarios}

Different scenarios of disruption are predicted based on the probabilities. As shown in figure 15, the input for the simulation of the first scenario is $40 \%$ probability of system-wide disruption and $60 \%$ probability of local damages. The input for the simulation for the second and third scenarios is $30 \%$ probability of system-wide disruption, in addition to $50 \%$ and $40 \%$ probability of local damages, respectively.

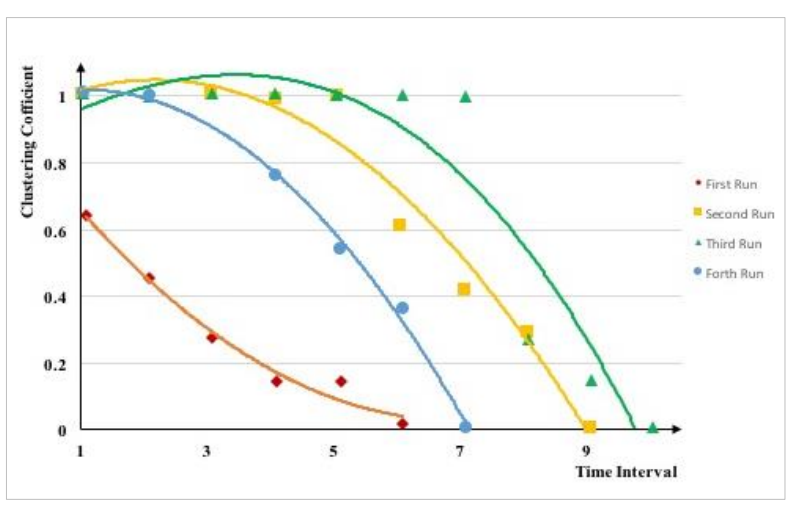

(a)

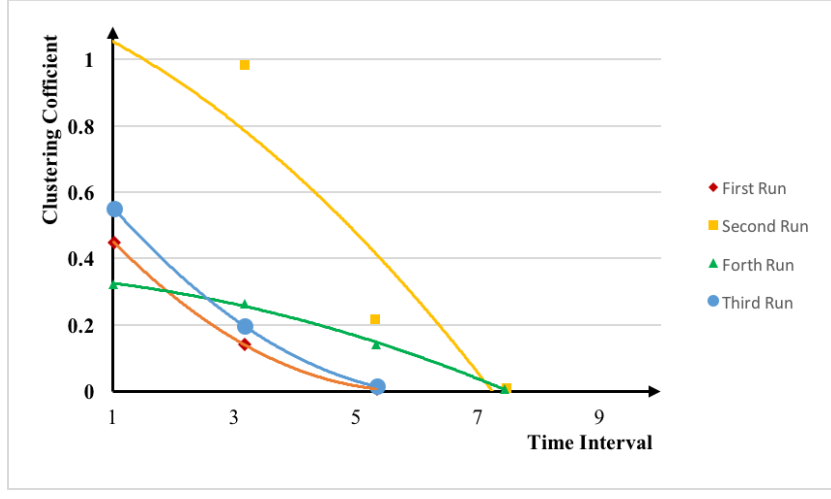

(b)

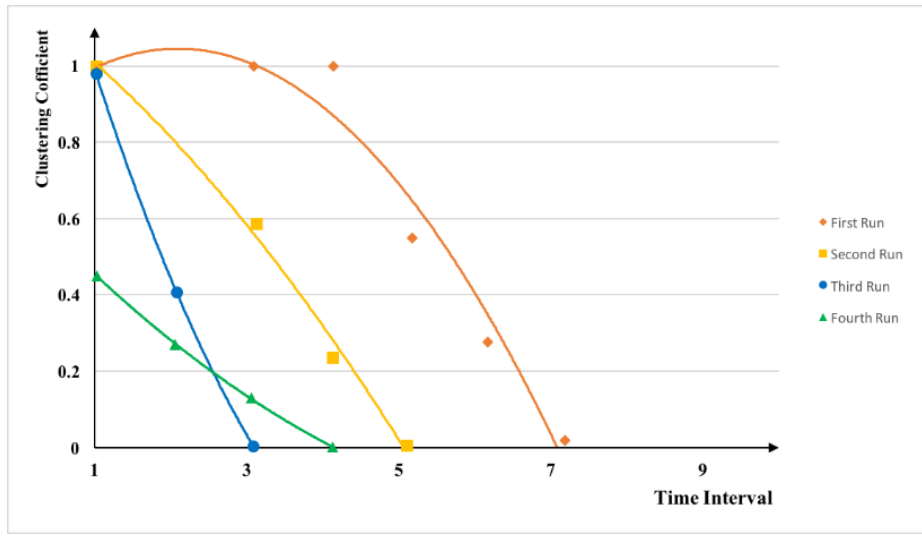

(c)

Figure 11. Variation of the clustering coefficient in numerous runs of simulation with: (a) $40 \%$ system-wide and $60 \%$ local disruption possibility over the time; (b) $30 \%$ system-wide and 50\% local disruption possibility over the time; (c) $30 \%$ system-wide and $40 \%$ local disruption possibility over the time 
Results of simulation shows that a rapid reduction of the clustering coefficient indicates more system-wide disruption of the system. The slope of the curve indicates whether disruption is system-wide or local. In this simulation, iterations in each run will stop, since the clustering coefficient becomes zero. In Figure 11, the trend line has been drawn for each run.

If the absolute value of the tangent to the curve is low, the damages are most likely local. If the absolute value is high, the damages are probably system-wide. The horizontal length from the origin, which demonstrates the time after disruption, is an index to measure the recoverability in the system. Possible patterns from the model are presented in this figure. Figure 11 a represents a system with a low capacity to recover and reconstitute service; thus, the system needs more time to obtain the serviceability of the infrastructure. From the observation of this figure, the recoverability in system-wide disruption is somehow higher than local disruption (Figure 11 a).

Figure $11 \mathrm{~b}$, c confirms that when the probability of local disruption increases, local disruption is manifested. In this situation, the entire system works, yet local damages cause disruption in the system. The figures present different possible patterns for disruption in the infrastructure systems with different features after the occurrence of any manmade or natural accident. Based on comparisons between the results of the simulations and the actual case study, four main categories for disruption in the infrastructure systems are identified.

(i) Low sensitivity and local disruption (Figure 12-curve 1)

(ii) High sensitivity and local disruption (Figure 12-curve 2)

(iii) High sensitivity and system-wide disruption (Figure 12-curve 3)

(iv) High sensitivity, system-wide and local disruption (Figure 12- curve 4)

These scenarios are drawn schematically, as followed Figure 12:

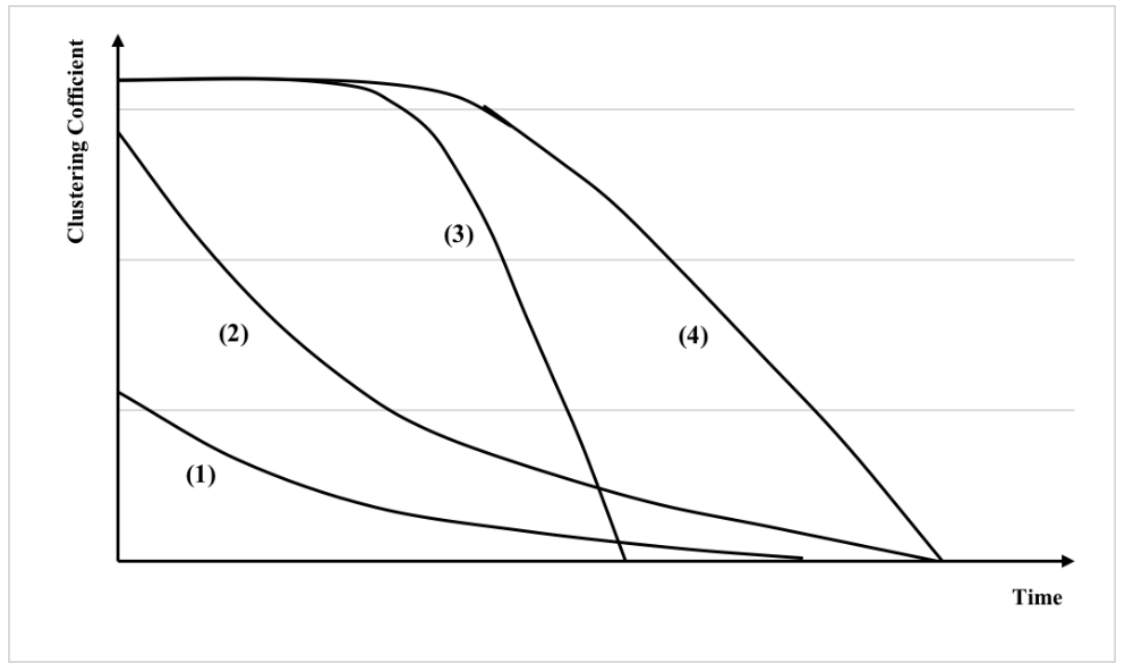

Figure 12. Possible system-wide versus local disruption patterns

\section{Data Collection and Case Study}

The development of urban areas is continuously increasing, which changes the complexity of the behavior of the infrastructures in a time of disaster. Kathmandu valley is one of the most populated areas near the epicenter of the earthquake. According to the studies, before the earthquake in 2015, the expectation was that Nepal would be extremely vulnerable to an earthquake due to rapid urbanization, lack of appropriate management and supervision in the development of the urban area, and poor maintenance and aging of infrastructures [37, 38].

Given the pivotal role that infrastructures have in daily life, understanding the behavior of the infrastructure systems would lead to more resilient systems. As vulnerability and resilience of the infrastructure are notably interconnected, in some cases, vulnerability is considered as one of the elements of assessing resilience. The vulnerability is measured using the collection of disruption data of the infrastructures to assess the resilience of the infrastructure systems. Based on the type of infrastructure, new technologies and devices is used to measure the deficiency in asphalt, power, and water infrastructures. It helps to understand environmental aspect and resiliency of the civil infrastructures [39].

Various approaches have been used to collect the data related to the infrastructures in the regions. Data in this paper has been collected from the Post-Disaster Need Assessment (PDNA) by the government of Nepal, reports from the Earthquake Engineering Research Institute (EERI), in-person interviews with international, national, and local agencies, and interviews with the local residents in the city of Bhaktapur. Since the data was gathered proportional to the number 
of inhabitants from population statistics and severity of damage in the Bhaktapur, the disruption data shows electric power outage throughout the entire city [40-42].

The moment magnitude of 7.8 earthquake on April 25, 2015 and several aftershocks struck the capital of Nepal, Kathmandu, and the surrounding areas. The infrastructures and buildings were severely damaged or destroyed and around 3.86 billion USD worth of assets were damaged. Based on the preliminary assessment, damage to infrastructures was estimated to be about 50 billion NRP (approximately 0.47 Billion USD). This devastating earthquake led to many electric power cuts, frequent disruption in the water system, and road blockages in the affected regions [20,36]. Studying these disruption in the aftermath of the earthquake provides a novel sight in the assessment of the characteristics and behavior of the CISs, despite existing load-shedding issue in the system.

In Bhaktapur, the infrastructure networks comprise a distance of 17 wards (i.e., boundaries of each small region of the city). The number of inhabitants varies between different wards and a large number of inhabitants are clustered in a few central wards. The households were randomly selected from the entire geographic region of the city to screen the pattern of infrastructure service disruption in the aftermath of the 2015 Gorkha earthquake. As is shown in figure 9 , the number of households who were interviewed, which in this paper is 22 , is proportional to the population density of the regions. Hence, samplings reflect the actual information of the infrastructure disruption throughout Bhaktapur. All data is collected via personal interviews around five months after the earthquake. The households surveyed are selected based on the severity of damages asked from local agent and residents [20, 36].

These data points are utilized to validate the output of the proposed model. In these samplings, a number of questions were asked about the damages to different infrastructures (such as water, electric power, and roads). The interviews provide useful data about the duration of the infrastructure service disruption in these households. Among the different infrastructure alternatives for the users, the electric power infrastructure is selected to verify the disruption-based model.

In the context of this study, homogeneity of the collected data regarding electricity infrastructures reduces the extra complexity and constraints in evaluation of the disruption pattern, which indicates the characteristics of the infrastructure network.

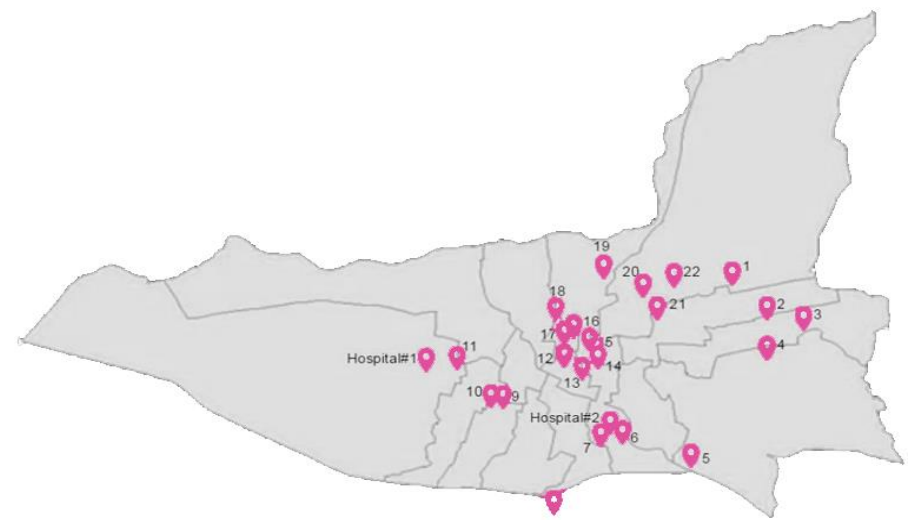

Figure 13. Sampling propagation between different wards in Bhaktapur after the earthquake [20]

The adjacency matrix provides some statistical characterizations of a network and defines the topologic layout of the network. The topology of the network displays the degree of coupling among the elements. Figure 14 shows the different networks at five different time points after the earthquake (i.e., just after the earthquake to 90 days - left to right respectively). Initially, most of the households have the same electricity disruption state and develop a network that is very dense in terms of network topology. Accordingly, this network has a higher clustering coefficient compared to the subsequent ones. With the passage of time after the earthquake, the number of edges in the networks decreases gradually, which implies the households regain their electricity service; therefore, the associated clustering coefficient of each network decreases. The network on the far right in Figure 12 is related to 90 days after the earthquake. The clustering coefficient of this network is equal to zero, because only two households are connected [44-45].
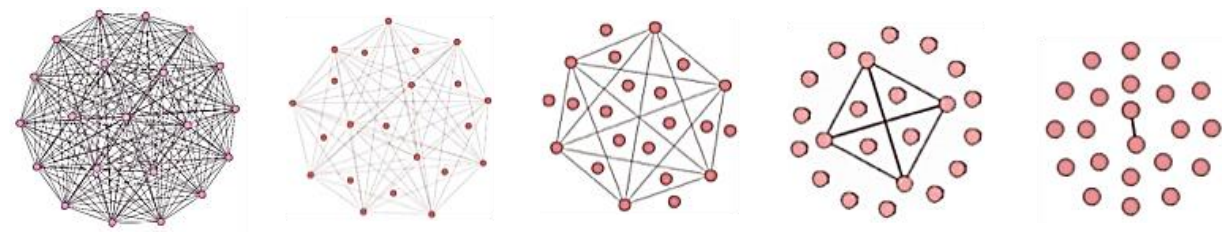

Figure 14. Dynamic changes in the disruption-based network at a different time from the baseline to the recovered situation (left to right) in the aftermath of the earthquake

In the next stage, all the cluster coefficients of the different networks are graphed over time of response, which is divided into 5-days intervals. Figure 15 shows the trend of variation in clustering coefficient. The required steps to create 
the graph are to identify similarity in the disruption state of the households (i.e., "peer"), obtain the adjacency matrices, develop different networks in a time division, obtain associated clustering coefficients, then plot these coefficients versus time. At the beginning, almost all the users did not have electricity after the earthquake, but electric power was gradually recovered.

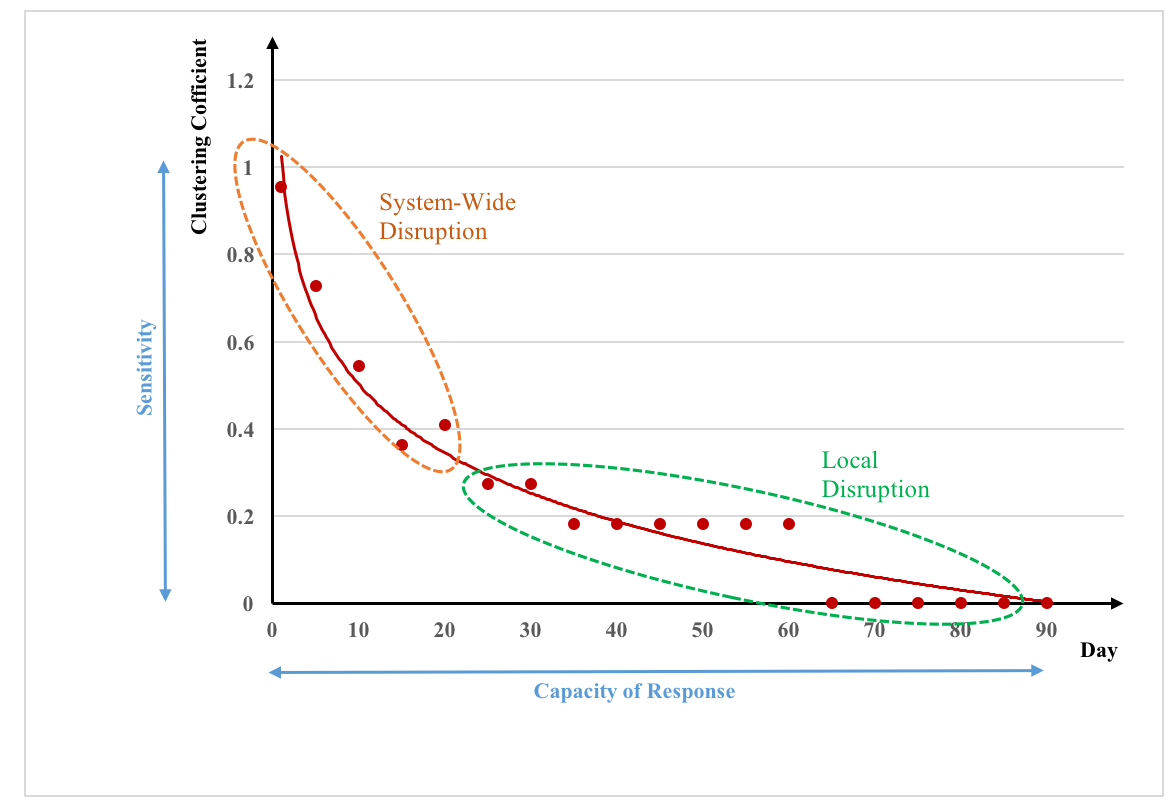

Figure 15. Variation of the clustering coefficient in electric power infrastructure in the case study of Nepal (Bhaktapur) over the time

Simulation of possible pattern needs a strong network and algorithm to capture the behavior of the system properly. The model can be used for further investigation on different network measures like modularity to understand: more about the structural topology of the network, the vulnerability of the clusters, or how to increase robustness of the network.

Three characterized results are retrieved from the model for the case study: (1) the amount of sensitivity in the beginning of the restoration process is almost high, (2) disruption patterns (local and system-wide) of the network are mostly local, and (3) recoverability of the electric power infrastructure in Bhaktapur is low. All these characteristics are shown in Figure 15, which depicts that the system is sensitive and system-wide disruption occurred within 25-30 days after the catastrophic event. Moreover, the damage in the power electric infrastructure was local and recovered slowly. Although, for the purpose of testing the proposed framework, one source of energy was considered as the sole provider of the power in Bhaktapur. In the case of large cities, whose energy is produced from different sources, this concern should be considered.

To conclude, lack of prior studies in the association between the resilience of the infrastructures in the system compels us to focus on disruption patterns in the infrastructure systems after a disaster. Peer notion, which has been widely used in social sciences, is applied to link the disrupted households and build the proposed model. Simulating various scenarios allows analysis of different types of disruption patterns in the infrastructure system that may occur in disasters such as the Nepal earthquake.

\section{Conclusion}

This work has highlighted the importance of service disruption in assessing several properties of a complex infrastructure network, particularly those related to resilience. The application of disruption data of end-users and social science has led to a novel network framework in characterizing of any CIS. An advantage of the proposed model is its simplicity, such that detailed information about the network would not be required.

In the modeling process at the peer level, different unknown end-users in different parts of the city are connected to each other based on similarities in disruption status (i.e., disruption period) of their service in the aftermath of a disaster. To this end, social networks associated to the infrastructure in each time interval are created and analyzed. Preliminary case study and simulations with the proposed algorithm show highly promising results in case of Bhaktapur city in Nepal. This algorithm is suited to be used in different CISs, such as water and transportation. Three main characteristics of infrastructure systems can be drawn from this approach and simulations. First, the sensitivity of the infrastructure networks relies on the clustering of the disrupted end-users and the tendency of nodes to be clustered together. A higher clustering coefficient indicates a higher level of system sensitivity after a disruption. Second, dropping the connectivity of a dense node compared to a less dense node leads to a major decrease in clustering coefficient and demonstrates a 
system-wide perturbation in the entire CIS and vice versa. Third, distance from the origin in time illustrates recoverability in the system. In addition, the result of the model reveals the pattern of disruption in the infrastructure network. The simulation evaluates the patterns of disruption in infrastructure networks that may occur in the aftermath of a disaster.

Initial findings emphasized the need for creating social networks based on more data points in the case study of Nepal. This study has proposed a novel methodological frame work and confirms this with the case study and simulations. In the case of Bhaktaput, the disruption seems more system wide and may be caused by the main sources, like power stations. This innovative framework opens a new chapter in the assessment of the resiliency of the communities and the future works will be able to focus on the contribution to this notion in the infrastructure systems and further evaluate this model with more data collected from other disasters. Furthermore, results of the simulations are consistent with the initial assumption of the associated figure. With increasing the probability of local disruption, the amount of the clustering coefficient obtained from related social network mainly decreases. Application of other measures of social network analysis is part of the future work of the authors in this study, which will open new windows toward the resilience of CISs.

This work can be further extended in several interesting directions. Firstly, the proposed network approach could be enhanced through the use of existing techniques in social network analyses and exploring the possibility of developing better algorithms for assessing the resilience of infrastructures with consideration of the disruption data and patterns [16]. Secondly, this method advances the concept of modeling physical civil infrastructure networks via numerous social networks and assesses the interdependency or dependency of the infrastructures and human-infrastructure coupling [35, 36]. Thirdly, based on the experimental information, the size of the data can be examined in the future to observe the impacts of the size on the accuracy of the model and to possibly find the threshold for this purpose. Finally, the proposed model creates the opportunity to bring new indices to network analyses, enabling them to evaluate the resilience concept, as well as fostering the potential of applying link prediction methods to evolve civil infrastructure networks that face perturbations and to increase their robustness.

\section{References}

[1] Barker, Kash, Jose Emmanuel Ramirez-Marquez, and Claudio M. Rocco. "Resilience-Based Network Component Importance Measures." Reliability Engineering \& System Safety 117 (September 2013): 89-97. doi:10.1016/j.ress.2013.03.012.

[2] Didier, Max, Marco Broccardo, Simona Esposito, and Bozidar Stojadinovic. "A Compositional Demand/supply Framework to Quantify the Resilience of Civil Infrastructure Systems (Re-CoDeS)." Sustainable and Resilient Infrastructure 3, no. 2 (September 12, 2017): 86-102. doi:10.1080/23789689.2017.1364560.

[3] Gay, Leon F., and Sunil K. Sinha. "Resilience of Civil Infrastructure Systems: Literature Review for Improved Asset Management." International Journal of Critical Infrastructures 9, no. 4 (2013): 330. doi:10.1504/ijcis.2013.058172.

[4] Gao, Jianxi, Baruch Barzel, and Albert-László Barabási. “Universal Resilience Patterns in Complex Networks.” Nature 530, no. 7590 (February 17, 2016): 307-312. doi:10.1038/nature16948.

[5] Zio, Enrico, Giovanni Sansavini, Roberto Maja, and Giovanna Marchionni. "An Analytical Approach to the Safety of Road Networks." International Journal of Reliability, Quality and Safety Engineering 15, no. 01 (February 2008): 67-76. doi:10.1142/s0218539308002939.

[6] Holmgren, Ake J. "Using Graph Models to Analyze the Vulnerability of Electric Power Networks." Risk Analysis 26, no. 4 (August 2006): 955-969. doi:10.1111/j.1539-6924.2006.00791.x.

[7] Hosseini, Seyedmohsen, Kash Barker, and Jose E. Ramirez-Marquez. "A Review of Definitions and Measures of System Resilience.” Reliability Engineering \& System Safety 145 (January 2016): 47-61. doi:10.1016/j.ress.2015.08.006.

[8] Porta, Sergio, Paolo Crucitti, and Vito Latora. “The Network Analysis of Urban Streets: A Dual Approach.” Physica A: Statistical Mechanics and Its Applications 369, no. 2 (September 2006): 853-866. doi:10.1016/j.physa.2005.12.063.

[9] Zhao, Kang, Akhil Kumar, Terry P. Harrison, and John Yen. “Analyzing the Resilience of Complex Supply Network Topologies Against Random and Targeted Disruptions.” IEEE Systems Journal 5, no. 1 (March 2011): 28-39. doi:10.1109/jsyst.2010.2100192.

[10] Masucci, A. P., D. Smith, A. Crooks, and M. Batty. "Random Planar Graphs and the London Street Network." The European Physical Journal B 71, no. 2 (August 18, 2009): 259-271. doi:10.1140/epjb/e2009-00290-4.

[11] Bompard, Ettore, Roberto Napoli, and Fei Xue. "Analysis of Structural Vulnerabilities in Power Transmission Grids." International Journal of Critical Infrastructure Protection 2, no. 1-2 (May 2009): 5-12. doi:10.1016/j.ijcip.2009.02.002.

[12] Crucitti, Paolo, Vito Latora, and Massimo Marchiori. "Locating critical lines in high-voltage electrical power grids." Fluctuation and Noise Letters 5, no. 02 (2005): L201-L208. doi:10.1142/s0219477505002562.

[13] Yazdani, A., and P. Jeffrey. "Applying Network Theory to Quantify the Redundancy and Structural Robustness of Water Distribution Systems." Journal of Water Resources Planning and Management 138, no. 2 (March 2012): 153-161. doi:10.1061/(asce)wr.1943-5452.0000159.

[14] Yazdani, Alireza, and Paul Jeffrey. "Water Distribution System Vulnerability Analysis Using Weighted and Directed Network 
Models.” Water Resources Research 48, no. 6 (June 2012). doi:10.1029/2012wr011897.

[15] Dueñas-Osorio, Leonardo, James I. Craig, and Barry J. Goodno. "Seismic Response of Critical Interdependent Networks." Earthquake Engineering \& Structural Dynamics 36, no. 2 (2007): 285-306. doi:10.1002/eqe.626.

[16] Liu, Yangyang, Chengli Zhao, Xiaojie Wang, Qiangjuan Huang, Xue Zhang, and Dongyun Yi. "The Degree-Related Clustering Coefficient and Its Application to Link Prediction.” Physica A: Statistical Mechanics and Its Applications 454 (July 2016): $24-33$. doi:10.1016/j.physa.2016.02.014.

[17] Reggiani, Aura, Peter Nijkamp, and Diego Lanzi. "Transport Resilience and Vulnerability: The Role of Connectivity." Transportation Research Part A: Policy and Practice 81 (November 2015): 4-15. doi:10.1016/j.tra.2014.12.012.

[18] Chai, C.-L., X. Liu, W.J. Zhang, and Z. Baber. "Application of Social Network Theory to Prioritizing Oil \& Gas Industries Protection in a Networked Critical Infrastructure System." Journal of Loss Prevention in the Process Industries 24, no. 5 (September 2011): 688-694. doi:10.1016/j.jlp.2011.05.011.

[19] Eisenberg, Daniel A., Jeryang Park, and Thomas P. Seager. "Sociotechnical Network Analysis for Power Grid Resilience in South Korea.” Complexity 2017 (2017): 1-14. doi:10.1155/2017/3597010.

[20] Nazarnia, H.; Mostafavi, A.; Pradhananga,N.; Ganapati, E. and R. Khanal, R. "Assessment of infrastructure resilience in developing Countries: a case study of water infrastructure in the 2015 Nepalese earthquake" Proceedings of the International Conference on Smart Infrastructure and Construction.

[21] Ouyang, Min. "Review on Modeling and Simulation of Interdependent Critical Infrastructure Systems." Reliability Engineering \& System Safety 121 (January 2014): 43-60. doi:10.1016/j.ress.2013.06.040.

[22] Hernandez-Fajardo, Isaac, and Leonardo Dueñas-Osorio. "Probabilistic Study of Cascading Failures in Complex Interdependent Lifeline Systems.” Reliability Engineering \& System Safety 111 (March 2013): 260-272. doi:10.1016/j.ress.2012.10.012.

[23] Sterbenz, James P. G., Egemen K. Çetinkaya, Mahmood A. Hameed, Abdul Jabbar, Shi Qian, and Justin P. Rohrer. "Evaluation of Network Resilience, Survivability, and Disruption Tolerance: Analysis, Topology Generation, Simulation, and Experimentation." Telecommunication Systems (December 7, 2011). doi:10.1007/s11235-011-9573-6.

[24] Hines, Paul, Eduardo Cotilla-Sanchez, and Seth Blumsack. "Do Topological Models Provide Good Information About Electricity Infrastructure Vulnerability?” Chaos: An Interdisciplinary Journal of Nonlinear Science 20, no. 3 (September 2010 ): 033122. doi:10.1063/1.3489887.

[25] Chang, Stephanie E., Timothy McDaniels, Jana Fox, Rajan Dhariwal, and Holly Longstaff. "Toward Disaster-Resilient Cities: Characterizing Resilience of Infrastructure Systems with Expert Judgments." Risk Analysis 34, no. 3 (October 23, 2013 ): 416-434. doi:10.1111/risa.12133.

[26] Chang, Stephanie E., Timothy McDaniels, Jana Fox, Rajan Dhariwal, and Holly Longstaff. "Toward Disaster-Resilient Cities: Characterizing Resilience of Infrastructure Systems with Expert Judgments.” Risk Analysis 34, no. 3 (October 23, 2013 ): 416-434. doi:10.1111/risa.12133.

[27] Winkler, James, Leonardo Dueñas-Osorio, Robert Stein, and Devika Subramanian. "Interface Network Models for Complex Urban Infrastructure Systems.” Journal of Infrastructure Systems 17, no. 4 (December 2011): 138-150. doi:10.1061/(asce)is.1943$555 x .0000068$.

[28] Meghanathan, Natarajan, Aniekan Essien, and Raven Lawrence. "A Two-Hop Neighbor Preference-Based Random Network Graph Model with High Clustering Coefficient for Modeling Real-World Complex Networks.” Egyptian Informatics Journal (September 2016). doi:10.1016/j.eij.2016.06.008.

[29] Wu, Zhihao, Youfang Lin, Jing Wang, and Steve Gregory. "Link Prediction with Node Clustering Coefficient." Physica A: Statistical Mechanics and Its Applications 452 (June 2016): 1-8. doi:10.1016/j.physa.2016.01.038.

[30] Adger, W. Neil. "Vulnerability." Global environmental change16, no. 3 (2006): 268-281.

[31] Gallopín, Gilberto C. "Linkages Between Vulnerability, Resilience, and Adaptive Capacity.” Global Environmental Change 16, no. 3 (August 2006): 293-303. doi:10.1016/j.gloenvcha.2006.02.004.

[32] Luers, Amy L. "The Surface of Vulnerability: An Analytical Framework for Examining Environmental Change." Global Environmental Change 15, no. 3 (October 2005): 214-223. doi:10.1016/j.gloenvcha.2005.04.003.

[33] Rinaldi, S.M., Peerenboom, J.P. and Kelly, T.K. "Identifying, Understanding, and Analyzing Critical Infrastructure Interdependencies.” IEEE Control Systems 21, no. 6 (December 2001): 11-25. doi:10.1109/37.96913[

[34] Bruneau, Michel, and Andrei Reinhorn. "Overview of the resilience concept." In Proceedings of the 8th US national conference on earthquake engineering, vol. 2040, pp. 18-22. 2006.

[35] Crucitti, Paolo, Vito Latora, and Massimo Marchiori. “A Topological Analysis of the Italian Electric Power Grid.” Physica A: Statistical Mechanics and Its Applications 338, no. 1-2 (July 2004): 92-97. doi:10.1016/j.physa.2004.02.029.

[36] Mostafavi, Ali, Nazife Emel Ganapati, Hadi Nazarnia, Nipesh Pradhananga, and Rewat Khanal. "Adaptive Capacity Under Chronic Stressors: Assessment of Water Infrastructure Resilience in 2015 Nepalese Earthquake Using a System Approach.” Natural Hazards Review 19, no. 1 (February 2018): 05017006. doi:10.1061/(asce)nh.1527-6996.0000263.

[37] Rasoulkhani, Kambiz, Maria Presa Reyes, and Ali Mostafavi. "Emergence of Resilience from Infrastructure Dynamics: A Simulation Framework for Theory Building.” Computing in Civil Engineering 2017 (June 13, 2017). 
doi:10.1061/9780784480847.032.

[38] Dixit, A. M. "Earthquake disaster risk management efforts in Nepal." In International Symposium Geohazards: Science, Engineering and Management. 2014.

[39] Baqersad, Mohamadtaqi, Mojtaba Mohammadafzali, Bouzid Choubane, Charles Holzschuher, Amirmasoud Hamedi, and Hesham Ali. "Precision Assessment of the Florida Texture Meter in Hot Mix Asphalt." Journal of Transportation Engineering, Part B: Pavements 144, no. 2 (June 2018): 04018003. doi:10.1061/jpeodx.0000029.

[40] Post Disaster Need Assessment (PDNA) (2015). Government of Nepal National Planning Commission. Available online: http://www.worldbank.org/content/dam/Worldbank/document/SAR/nepal/PDNA\%20Volume\%20A\%20Final.pdf, (Accessed in August 2015).

[41] Kaushik, Hemant, John Bevington, Kishor Jaiswal, Bret Lizundia, and Surya Shrestha. Buildings (EERI Earthquake Reconnaissance Team Report: M7. 8 Gorkha, Nepal Earthquake on April 25, 2015 and its Aftershocks). Earthquake Engineering Research Institute, 2016.

[42] Uprety, Sital, Juliet Iwelunmor, Nora Sadik, Bipin Dangol, and Thanh H. Nguyen. "A Qualitative Case Study of Water, Sanitation, and Hygiene Resources after the 2015 Gorkha, Nepal, Earthquake.” Earthquake Spectra 33, no. S1 (December 2017): S133-S146. doi:10.1193/112916eqs212m.

[43] Ennett, Susan T., and Karl E. Bauman. "Peer Group Structure and Adolescent Cigarette Smoking: A Social Network Analysis." Journal of Health and Social Behavior 34, no. 3 (September 1993): 226. doi:10.2307/2137204.

[44] Boccaletti, Stefano, Vito Latora, Yamir Moreno, Martin Chavez, and D-U. Hwang. "Complex networks: Structure and dynamics." Physics reports 424, no. 4-5 (2006): 175-308. doi:10.1016/j.physrep.2005.10.009.

[45] Newman, M. E. J. “The Structure and Function of Complex Networks.” SIAM Review 45, no. 2 (January 2003 ): $167-256$. doi:10.1137/s003614450342480.

[46] Clauset, Aaron, Cristopher Moore, and M. E. J. Newman. "Hierarchical Structure and the Prediction of Missing Links in Networks." Nature 453, no. 7191 (May 2008): 98-101. doi:10.1038/nature06830. 